
\title{
25 Research Soure \\ Reduced Brain Volume and White Matter Alterations in Shank3-Deficient Rats
}

Carla Esther Meyer Golden

New York University https://orcid.org/0000-0003-1445-3724

Victoria X Wang

Icahn School of Medicine at Mount Sinai

\section{Hala Harony-Nicolas}

Icahn School of Medicine at Mount Sinai Department of Psychiatry

Patrick R. Hof

Icahn School of Medicine at Mount Sinai Friedman Brain Institute

Joseph Buxbaum ( $\sim$ joseph.buxbaum@mssm.edu )

Icahn School of Medicine at Mount Sinai https://orcid.org/0000-0001-8898-8313

\section{Research}

Keywords: Shank3, magnetic resonance imaging, diffusion tensor imaging, superior and inferior colliculus

Posted Date: November 4th, 2020

DOl: https://doi.org/10.21203/rs.3.rs-100329/v1

License: (c) (i) This work is licensed under a Creative Commons Attribution 4.0 International License. Read Full License

Version of Record: A version of this preprint was published at Autism Research on July 27th, 2021. See the published version at https://doi.org/10.1002/aur.2568. 


\section{Abstract}

Background: Mutations and deletions in the SHANK3 synaptic gene cause the major neurodevelopmental features of Phelan-McDermid syndrome (PMS). The SHANK3 gene encodes a key structural component of excitatory synapses that is important for synaptogenesis. PMS is characterized by intellectual disability, autism spectrum disorder, cognitive deficits, physical dysmorphic features, sensory hyporeactivity, and alterations in the size of multiple brain regions. Clinical assessments and limited imaging studies have revealed a reduction in volume of multiple brain regions. They have also found white matter thinning and microstructural alterations to be persistent in patients with PMS. While many of these impairments have been replicated in mouse models of PMS, the brain structure of a rat model has not yet been studied.

Methods: We assessed the brain structure of haploinsufficient and homozygous Shank3-deficient rats that model the behavioral deficits of PMS with magnetic resonance and diffusion tensor imaging, and compared their brain structure to wild type littermates.

Results: Both gray and white matter structures were smaller in Shank3-deficient rats, leading to an overall reduction in brain size compared to wild type littermates. The largest region to be diminished in size was the neocortex. Some regions involved in sensory processing and white matter regions were also reduced in size. Lastly, the microstructure of two white matter tracts, the external capsule and fornix, was abnormal.

Conclusions: Shank3-deficient rats replicate the reduced brain volume and altered white matter phenotypes present in individuals with PMS. Therefore, the brain regions that were altered represent potential cross-species structural biomarkers that warrant further study.

\section{Background}

Phelan-McDermid syndrome (PMS) is a rare neurodevelopmental disorder caused by deletions of, or point mutations within, the terminal chromosomal region 22q13.3 that encompasses the SHANK3 gene. The characteristics of PMS include intellectual disability, autism spectrum disorder (ASD), attention deficits, impaired communication, motor abnormalities, seizures, recurrent medical comorbidities, neonatal hypotonia, and macrocephaly (1). Additionally, alterations in reactivity to sensory stimuli is frequently observed in PMS $(2,3)$. The neuroanatomical abnormalities that could be underlying these physical and behavioral phenotypes have gone largely understudied. Understanding how the pathology that results from a Shank3 deficiency affects brain structure in PMS pathogenesis could offer biomarkers to focus on in patients and rodent models. Neuroimaging studies on small cohorts have shown that individuals with PMS have reduced relative volumes of structures in the basal ganglia and cerebellum $(4,5)$, white matter thinning $(6,7)$, and alterations in fiber tracts $(8,9)$. However, these abnormalities have not yet been explored in a rat model. Rats offer larger brains than mice and thus finer imaging resolution as well as homology that is closer to humans compared to mice. 
We used haploinsufficient and homozygous knockout (KO) rat models of PMS that we have previously demonstrated have social memory and attention deficits in addition to impaired synaptic plasticity (10) to investigate the effect of Shank3 deficiency on brain structure with MRI and DTI.

\section{Methods}

\section{Animal care and husbandry}

This study used male wild type (WT), Shank $3^{+/}$, and Shank $3^{-/}$littermate three-month-old rats. Shank3deficient rats were generated using zinc-finger nucleases on the outbred Sprague-Dawley background, as previously described (10). All rats were kept under veterinary supervision in a $12 \mathrm{~h}$ reverse light/dark cycle at $22 \pm 2^{\circ} \mathrm{C}$. Animals were pair-caged with food and water available ad libitum. All animal procedures were approved by the Institutional Animal Care and Use Committee at the Icahn School of Medicine at Mount Sinai.

\section{MRI}

All imaging was performed by the BioMedical Molecular Imaging Institute using a Bruker Biospec 70/30 7 T scanner with a B-GA12S gradient insert (gradient strength $440 \mathrm{mT} / \mathrm{m}$ and slew rate $3444 \mathrm{~T} / \mathrm{m} / \mathrm{s}$ ). A Bruker 4-channel rat brain phased array was used for all data acquisition in conjunction with a Bruker volume transmit $86-\mathrm{cm}$ coil. All rats were imaged on a heated bed and respiration was monitored continuously until the end of the scan. The animals were anesthetized using isoflurane anesthesia ( $3 \%$ induction and $1.5 \%$ maintenance). After a three-plane localizer, a field map was acquired and the rat brain was shimmed using Mapshim software. A DTI protocol was acquired with a Pulsed Gradient Spin Echo Echo-planar imaging (EPI) sequence with the following parameters: repetition time $(T R)=5000 \mathrm{~ms}$, echo time $(T E)=22.6 \mathrm{~ms}$, 4 segments, 30 gradient directions with b-value $=1000 \mathrm{~s} / \mathrm{mm}^{2}$ and 5 BO's, field of view $(\mathrm{FOV})=25 \mathrm{~mm}$, matrix $=128 \times 128$, slice thickness $=1 \mathrm{~mm}$, skip $=0,6$ averages, total acquisition time $=1 \mathrm{hr}$. The voxel size was $0.195 \times 0.195 \times 1 \mathrm{~mm}^{3}(1000 \mu \mathrm{m}$-thick). A high resolution T2 anatomical scan was obtained with a 3D Rapid Acquisition with Relaxation Enhancement (RARE) sequence with a RARE factor of $8, T R=777 \mathrm{~ms}$, effective TE $=52 \mathrm{~ms}$, FOV $=30 \mathrm{~mm} \times 27.25 \mathrm{~mm} \times 30 \mathrm{~mm}$, matrix size 256 x 256 x 128. The voxel size was $0.117 \times 0.117 \times 0.234 \mathrm{~mm}^{3}$ ( $234 \mu \mathrm{m}$-thick).

\section{MRI region-based analytical pipeline with manual editing}

A magnetic resonance imaging processing pipeline was used to perform semi-automated nonbiased

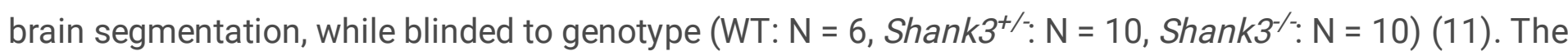
pipeline is composed of six major steps: rigid registration of images to each other, generation of a wholebrain mask for each image, averaging of all images, creation of a whole-brain mask for this averaged image, segmentation of the average mask by regions of interest (ROIs), parcellation propagation of the segmented mask to individual subjects, and ROI-based statistics for the individual images. The deformation necessary to warp each subject's image to the average was used to calculate the volume of 
the ROls. After each mask was generated, it was improved manually in ITK-SNAP (www.itksnap.org). The segmentation into ROls was determined by a template that was hand-segmented into 32 brain regions, listed in Supplementary Table 1.

Automatically generated segmented masks for the individual images that did not closely match the average segmented mask (two WT and one Shank $3^{-/} \mathrm{T} 2$ mask) and individual data point outliers, defined as being outside 1.5 times the interquartile range, were excluded from the analysis. The whole-brain masks were used to determine whole-brain measures. Mean voxel intensity for each ROI and across the whole-brain was measured in both the T2 and DTI images and the volume of each ROI and whole-brain was calculated from the T2 images. Only white matter structures were included in the DTI analysis (see Supplementary Table 1). For each independent variable, if the distribution was nonparametric according to the Shapiro-Wilk's test, a Kruskal-Wallis test was performed, and if the distribution was normally distributed, a two-way ANOVA was used. Due to the fact that many comparisons were made across ROIs, the output was then assessed for the ability to survive a correction for multiple comparisons with a Bonferroni correction. Genotype was the only between-groups factor. If an effect had a significant nominal $p$-value (0.05), pairwise comparisons were made. If the data were parametric, a Tukey HSD test was used to compare the individual means. If the data were nonparametric, a Dunn's test was used for pairwise comparisons. An adjustment of the $p$-values was made to account for the additional comparisons. The effect size of each volumetric change was measured with a Cohen's $d$. Custom scripts written in the R statistical programming environment were used for the statistical analyses $(R$ Development Core Team, 2006).

\section{Results}

\section{Shank3 deficiency results in reduced brain volume}

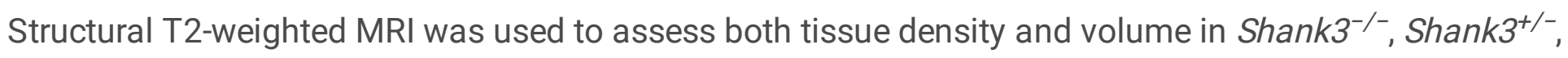
and WT littermates across the whole brain and in regions of interest (ROIs) (see all results in

Supplementary Table 2). Notably, none of the nominal $p$-values from the pairwise comparisons survived a Bonferroni correction. Remarkably, we found a significant effect of genotype on the volume of the whole brain (Fig. 1A and B; ANOVA: $p=0.049$ ), the brains of the Shank $3^{-/-}$rats showing a smaller size compared to their Shank $3^{+/-}$and WT littermates. This reduction in overall brain volume was driven by a decrease in the absolute volume of larger gray matter brain structures in the Shank $3^{-/-}$rats, including the neocortex, the piriform cortex, the thalamus, and the rest of forebrain (the regions of the forebrain that were not otherwise included in the list of ROIs) (Fig. 1A and B; ANOVA followed by Tukey HSD: Shank3 $3^{+/-}$

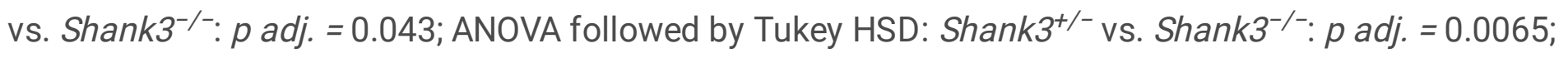

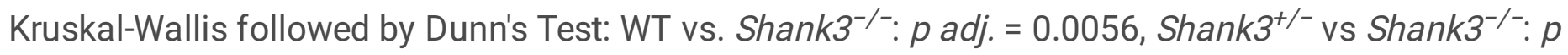

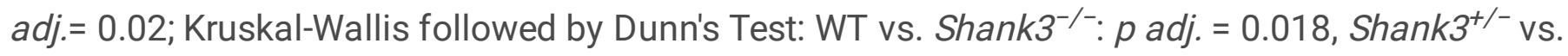
Shank3 ${ }^{-/-}: p$ adj. $\left.=0.012\right)$. The inferior and superior colliculi also had significantly smaller absolute

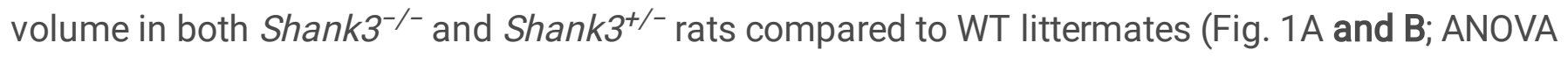




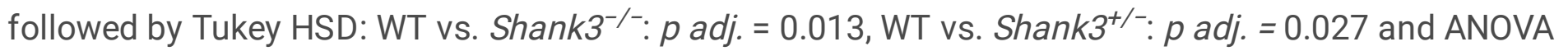

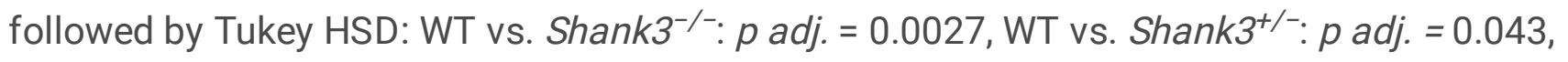
respectively). Finally, two white matter structures, the internal capsule and anterior commissure, were also

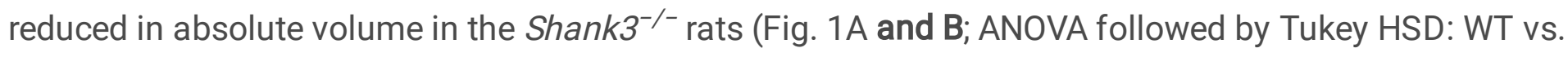

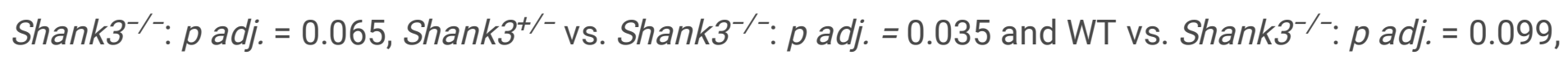

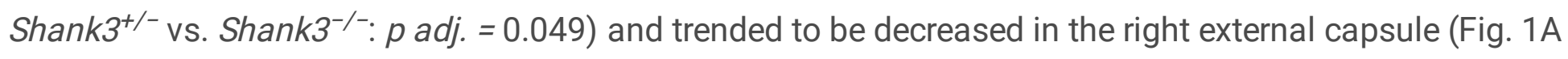
and B; ANOVA: $p=0.085$ ). The effect sizes of all changes were large (Cohen's $d>|0.8|$ ). Tissue density was not significantly altered and no alterations were identified in the volume of the ventricular system.

We next examined whether any ROls were also altered in relative volume by calculating their percentage of the total brain volume. Due to the fact that the entire brain decreased in volume in Shank $3^{-/-}$rats, many of the brain regions that were decreased in absolute volume were not also decreased in relative volume. The only brain region that had a significantly lower relative volume in Shank $3^{-/-}$rats compared to WT littermates was the superior colliculus (Fig. 1A and C; ANOVA followed by Tukey HSD: WT vs.

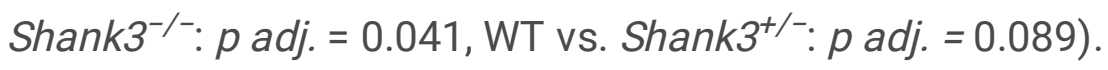

\section{Loss of Shank3 expression leads to deficits in integrity of white matter regions}

Lastly, we used DTI to assess the integrity of the white matter that connects these regions. Four indices were calculated from these images and used to describe the diffusion: axial, radial, and mean diffusion $(A D, R D$, and $M D)$ and fractional anisotropy (FA). We examined diffusion in nine white matter pathways in Shank3-deficient rats and compared them to their WT littermates (see all results in Supplementary Table 2). We found that the nominal $p$-values for the DTI metrics of some of these regions were significant, however, none of them survived a Bonferroni correction. AD was significantly reduced in the

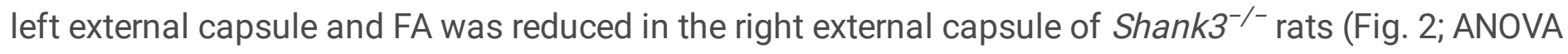

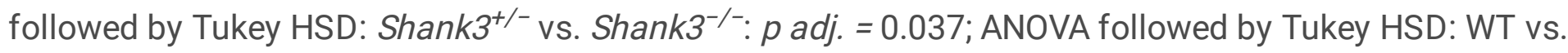

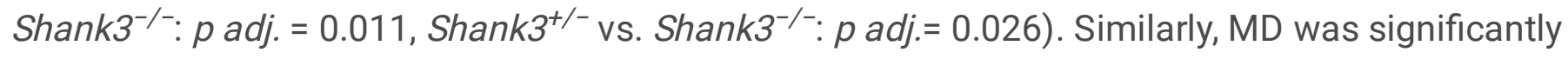
increased in the fornix in Shank $3^{-/-}$rats compared to WT littermates (Fig. 2; ANOVA followed by Tukey HSD: WT vs. Shank3 $\left.{ }^{-/-}: p=0.04\right)$.

\section{Discussion}

In this study, we discovered structural perturbations in the brains of Shank3-deficient rats, including an overall reduction in brain volume that was driven by a decrease in the volume of large, gray matter regions and alterations in the structural integrity of white matter domains.

Limited studies have shown that Shank3 deficiency leads to decreased brain volumes in humans as well. A study of eleven individuals with PMS found reduced relative volumes of caudate nucleus, putamen, left pallidum, and cerebellar vermis (4). In a separate study, ten patients with a 22q13 terminal deletion had 
cerebellar vermis hypoplasia (5). It would be worthwhile to explore the cause of this reduction in regional volumes in rodent models. At the cellular level, Shank3 protein deficiency in Shank3 ${ }^{e 4-9}$ and Shank3B ${ }^{-/-}$ mice has been shown to affect expression of synaptic proteins, spine head length, glutamatergic postsynaptic densities, dendritic arborizations and length, and spine density $(12,13)$. Shank $3 B^{-/-}$mice have reduced levels of SAPAP3, Homer-1b/c, and PSD-93 in the postsynaptic densities of their striatum, a reduction in the glutamate receptor subunits GluR2, NR2A and NR2B, significantly reduced mean thickness and length of postsynaptic densities in glutamatergic neurons, and reduced spine densities of medium spiny neurons (13). Shank3 ${ }^{e 4-9}$ mice also have reduced expression of synaptic proteins (12). Future studies should examine how these cellular phenotypes affect the structure of these brain regions.

Many individuals with PMS are hyporeactive to sensory stimuli $(2,3)$. The superior and inferior colliculus are part of the visual and auditory pathways, respectively, and the thalamus plays a major role in relaying peripheric sensory information to the cerebral cortex. All three regions were smaller in Shank3-deficient rats. The response of the auditory cortex of Shank $3^{+/-}$rats to speech and non-speech sounds has been shown to be weaker than WT controls, with less spontaneous firing (14). Sensory sensitivities are a core feature of ASD (15). Interestingly, we recently reported comparable alterations in a rat model of another monogenic cause of ASD, Fragile $X$ syndrome, using the same imaging techniques and the same parameters (16), indicating a commonality of sensory deficits between syndromes. The structure of these regions should be studied in relation to these functional consequences in rodent models of PMS.

The internal capsule and anterior commissure were decreased in absolute volume, AD was reduced in the external capsule, and MD was increased in the fornix of Shank3-deficient rats. These DTI findings suggest that white matter microstructure is not properly able to restrict diffusion along the axis of the axonal fibers, which may functionally affect major corticocortical and corticosubcortical projections. The fornix is one of the major output pathways of the hippocampus, where both long-term potentiation and mGluR-dependent long-term depression are impaired in these rats (10). Future studies should determine whether the deficits in white matter microstructure have any bearing on the impairments in activity.

These white matter deficits parallel those seen in humans and a mouse model. Clinical assessment and studies of individuals with Shank3 deficiency, 22q13.3 deletion syndrome, and diagnosed PMS showed thinning or hypoplasia of the corpus callosum $(6,7)$ and reduced FA and increased MD in long association fiber tracts $(8,9)$, including the uncinate fasciculus and the inferior fronto-occipital fasciculus. Similarly, Shank3 heterozygous KO mice have significantly reduced FA in long fiber tract systems compared to WT controls (8). Therefore, our study supports others in their conclusion that Shank3 deficiency leads to white matter disease. It also lays the groundwork for future studies to examine the relationship between these anatomical impairments and the behavioral deficits we have previously identified in these Shank3-deficient rats (10).

\section{Limitations}


The generalizability of our findings is limited to males because we did not include female rat models of PMS. Future rodent studies should explore whether these impairments vary by sex. A further potential limitation of the current study is that whereas we had 10 animals per Shank3-deificient rat group, we only included imaging data from 6 WT rats. This was due to our main focus being on collecting data from littermates so that we could account for extraneous factors that were not relevant to the study, like parents, birth time, age, etc. We were therefore limited by the amount of variability in genotype in each litter. Lastly, many of our findings were not significant after correcting for multiple comparisons. For this reason, in addition to supplying the nominal $p$ values, we have reported whether they survived this correction or not.

\section{Conclusions}

Our MRI and DTI findings indicate that Shank3-deficiency in rats leads to reduce brain volumes and impaired white matter pathways. The colliculi were among the regions that were affected, suggesting a potential locus for the sensory deficits often documented in PMS. Both reduced volume and white matter impairment have been identified in humans and mouse models with Shank3-deficiency, suggesting that they are potential cross-species structural biomarkers that warrant further study.

\section{List Of Abbreviations}

PMS = Phelan McDermid syndrome

ASD $=$ Autism spectrum disorder

$\mathrm{KO}=\mathrm{Knock}$ out

$\mathrm{MRI}=$ Magnetic resonance imaging

$\mathrm{DTI}=$ Diffusion tensor imaging

WT = Wild type

$\mathrm{ROI}=$ Regions of interest

$\mathrm{EPI}=$ Echo-planar imaging

$\mathrm{TR}=$ Repetition time

$\mathrm{TE}=$ Echo time

FOV $=$ Field of view

RARE $=$ Rapid acquisition with relaxation enhancement 
$A D=$ Axial diffusion

$\mathrm{RD}=$ Radial diffusion

$M D=$ Mean diffusion

$\mathrm{FA}=$ Fractional anisotropy

\section{Declarations}

\section{Availability of data and materials}

The datasets used and analyzed during the current study are available from the corresponding author on reasonable request.

\section{Competing interests}

The authors declare that they have no competing interests

\section{Funding}

Seaver Foundation (to JDB, HHN, and CEMG); Autism Speaks (to JDB) and the National Institute of Mental Health (F31 MH115656-01 to CEMG and 5R01 MH101584-05 to JDB). CEMG was a Seaver Graduate Fellow at the time of this study.

\section{Author's contributions}

CEMG and JDB designed the study. VXW acquired the imaging data. CEMG analyzed and interpreted the data, and wrote the paper. PRH and HHN helped interpret the data and revise the manuscript. All authors read and approved the final manuscript.

\section{Acknowledgements}

We thank Emma Huang, Olamide Olawuni, Lyaba Mahmood, Jackson Novick, and Alice Cheng, who contributed to the analysis of the data. We also thank the BioMedical Molecular Imaging Institute for their services in carrying out the magnetic resonance imaging.

\section{References}

1. Harony-Nicolas H, De Rubeis S, Kolevzon A, Buxbaum JD. Phelan McDermid Syndrome: From Genetic Discoveries to Animal Models and Treatment. J Child Neurol. 2015;30(14):1861-70.

2. Droogmans G, Swillen A, Van Buggenhout G. Deep Phenotyping of Development, Communication and Behaviour in Phelan-McDermid Syndrome. Mol Syndromol. 2020;10(6):294-305. 
3. De Rubeis S, Siper PM, Durkin A, Weissman J, Muratet F, Halpern D, et al. Delineation of the genetic and clinical spectrum of Phelan-McDermid syndrome caused by SHANK3 point mutations. Mol Autism. 2018;9:31.

4. Srivastava S, Scherrer B, Prohl AK, Filip-Dhima R, Kapur K, Kolevzon A, et al. Volumetric Analysis of the Basal Ganglia and Cerebellar Structures in Patients with Phelan-McDermid Syndrome. Pediatr Neurol. 2019;90:37-43.

5. Aldinger KA, Kogan J, Kimonis V, Fernandez B, Horn D, Klopocki E, et al. Cerebellar and posterior fossa malformations in patients with autism-associated chromosome 22q13 terminal deletion. Am J Med Genet A. 2013;161A(1):131-6.

6. Soorya L, Kolevzon A, Zweifach J, Lim T, Dobry Y, Schwartz L, et al. Prospective investigation of autism and genotype-phenotype correlations in 22q13 deletion syndrome and SHANK3 deficiency. Mol Autism. 2013;4(1):18.

7. Philippe A, Boddaert N, Vaivre-Douret L, Robel L, Danon-Boileau L, Malan V, et al. Neurobehavioral profile and brain imaging study of the 22q13.3 deletion syndrome in childhood. Pediatrics. 2008;122(2):e376-82.

8. Jesse S, Muller HP, Schoen M, Asoglu H, Bockmann J, Huppertz HJ, et al. Severe white matter damage in SHANK3 deficiency: a human and translational study. Ann Clin Transl Neurol. 2020;7(1):46-58.

9. Bassell J, Srivastava S, Prohl AK, Scherrer B, Kapur K, Filip-Dhima R, et al. Diffusion Tensor Imaging Abnormalities in the Uncinate Fasciculus and Inferior Longitudinal Fasciculus in Phelan-McDermid Syndrome. Pediatr Neurol. 2020;106:24-31.

10. Harony-Nicolas H, Kay M, du Hoffmann J, Klein ME, Bozdagi-Gunal O, Riad M, et al. Oxytocin improves behavioral and electrophysiological deficits in a novel Shank3-deficient rat. Elife. 2017;6.

11. Budin F, Hoogstoel M, Reynolds P, Grauer M, O'Leary-Moore SK, Oguz I. Fully automated rodent brain MR image processing pipeline on a Midas server: from acquired images to region-based statistics. Front Neuroinform. 2013;7:15.

12. Wang X, McCoy PA, Rodriguiz RM, Pan Y, Je HS, Roberts AC, et al. Synaptic dysfunction and abnormal behaviors in mice lacking major isoforms of Shank3. Hum Mol Genet. 2011;20(15):3093108.

13. Peca J, Feliciano C, Ting JT, Wang W, Wells MF, Venkatraman TN, et al. Shank3 mutant mice display autistic-like behaviours and striatal dysfunction. Nature. 2011;472(7344):437-42.

14. Engineer CT, Rahebi KC, Borland MS, Buell EP, Im KW, Wilson LG, et al. Shank3-deficient rats exhibit degraded cortical responses to sound. Autism Res. 2018;11(1):59-68.

15. Posar A, Visconti P. Sensory abnormalities in children with autism spectrum disorder. J Pediatr (Rio J). 2018;94(4):342-50.

16. Golden CEM, Yee Y, Wang VX, Harony-Nicolas H, Hof PR, Lerch JP, et al. Reduced axonal caliber and structural changes in a rat model of Fragile $X$ syndrome with a deletion of a K-Homology domain of Fmr1. Transl Psychiatry. 2020;10(1):280. 


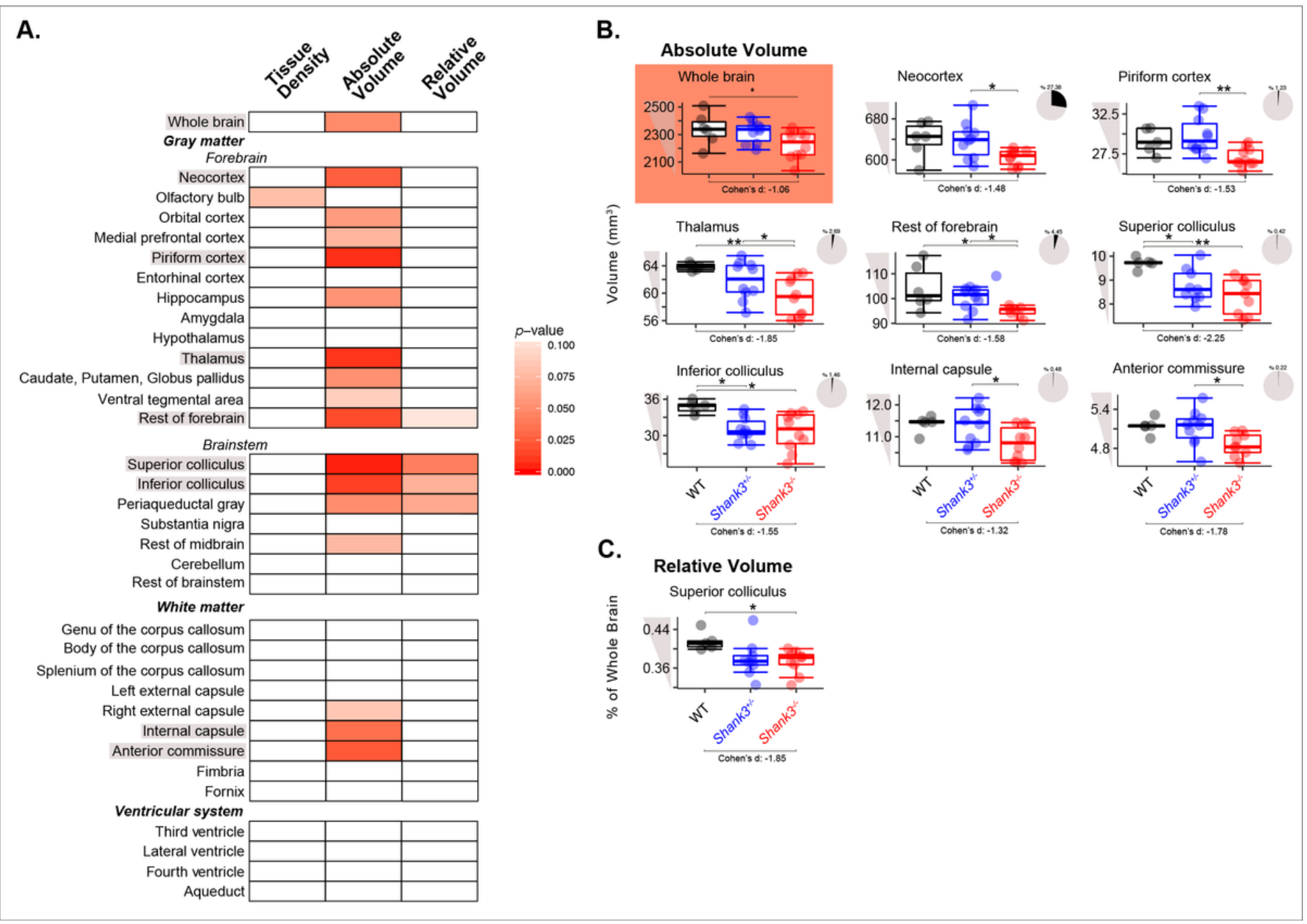

\section{Figure 1}

Tissue density and absolute and relative volume of brain regions of WT and Shank3-deficient littermates using T2 MRI. (A) Heatmap of p-values from ANOVA and Kruskal-Wallis tests for an effect of genotype on tissue density and absolute and relative volumes where an increase in red denotes an increase in significance of nominal p-values. Boxplots of the brain regions that significantly changed in (C) absolute and (D) relative volume. Effect sizes for the differences between WT and Shank3-/- littermates were reported as Cohen's $d$ below the graphs and are considered large if they are $>|0.8|$. The pie charts for each absolute volume represent the percent of the total volume each region comprises in the WT rats. Significance bars represent the effect of genotype or pair-wise comparisons from a Tukey HSD or Dunn's test if they were significant after an effect of genotype was significant, (WT: $N=6$, Shank3+/-: $N=10$, Shank3-/-: $N=10),{ }^{\star *} p<0.01,{ }^{*} p<0.05$. 


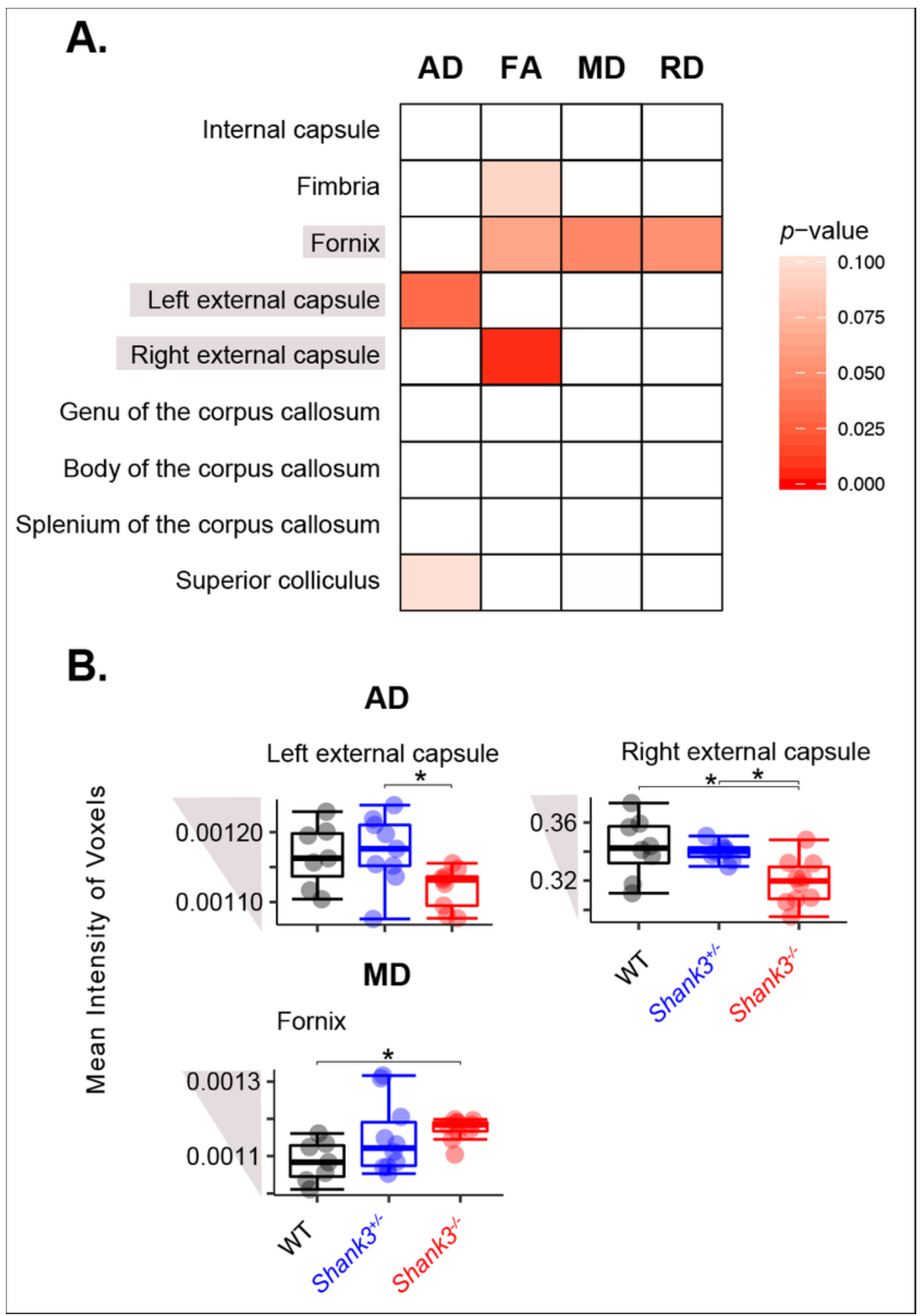

Figure 2

Changes in diffusion indices of WT and Shank3-deficient littermates using DTI. (A) Heatmap of p-values from ANOVA and Kruskal-Wallis tests for the effect of genotype on AD, FA, MD, and RD where an increase in red denotes increased significance. (B) Boxplots of mean intensity of voxels across altered regions in Shank3-deficient rats. Significance bars represent pair-wise comparisons from a Tukey HSD test after an effect of genotype was significant, (WT: $N=6$, Shank3+/-: $N=10$, Shank3-/-: $N=10)$, *p $<0.05$, \#p $<0.1$. 


\section{Supplementary Files}

This is a list of supplementary files associated with this preprint. Click to download.

- SupplementaryTable1.xIsx

- SupplementaryTable2.xlsx 